\title{
Orai1: CRACing the Th17 response in AKI
}

\author{
Sanjeev Noel
}

Division of Nephrology, Department of Medicine, Johns Hopkins University, Baltimore, Maryland, USA.

\begin{abstract}
A strong Th17 inflammatory response aggravates ischemia reperfusioninduced (IR-induced) acute kidney injury (AKI), tissue fibrosis, and AKI-to-chronic kidney disease (CKD) progression. However, the underlying mechanisms of sustained Th17 activation following AKI and during AKIto-CKD progression are unclear. In this issue of the $J C I$, Mehrotra et al. present compelling evidence that the store-operated calcium $\left(\mathrm{Ca}^{2+}\right)$ channel Orai1 sustains Th17-driven inflammatory response after AKI and drives the AKI-to-CKD transition. Orai1 blockade significantly protected renal function from IR, attenuated high-salt-induced AKI-to-CKD progression in rats, and decreased Th17 response in rat and human T cells. Therapeutic targeting of Orai1 can potentially reduce AKI, AKI-to-CKD progression, and other Th17driven diseases.
\end{abstract}

\section{Th17 cells in AKI}

Acute kidney injury (AKI) affects 2\%-5\% of hospitalized patients and increases the risk of adverse long-term effects, such as development of chronic kidney disease (CKD) and progression to end-stage renal disease. Additionally, AKI occurs in more than $50 \%$ of intensive care unit (ICU) patients (1). There is a complex interplay between multiple pathophysiologic mechanisms, including recruitment and activation of immune cells, which drives AKI pathogenesis $(2,3)$. T cells were first implicated in the pathophysiology of AKI approximately two decades ago (4), and follow-up studies identified $\mathrm{CD}^{+} \mathrm{T}$ cells as the major pathogenic $\mathrm{T}$ cell subset (5). Broadly, $\mathrm{CD}^{+} \mathrm{T}$ cells differentiate into proinflammatory Th1 cells $\left(\mathrm{IFN}-\gamma^{+} \mathrm{Tbet}^{+}\right)$ and Th17 cells (IL-17 ${ }^{+}$, ROR $\left.\gamma \mathrm{T}^{+}\right)$, or antiinflammatory Th2 cells (IL- $4^{+}$, GATA $^{+}$) and Tregs $\left(\mathrm{IL}-10^{+}, \mathrm{FOXP}^{+}\right)$, depending on the type of the initial insult and the cytokine microenvironment in the injured/ infected tissue. Many other Th cells such as Th9, Th22, and T follicular helper (Tfh) have also been identified (6).
Ischemia reperfusion (IR) injury, the most common cause of AKI, is believed to predominantly elicit a Th1 proinflammatory response (7). However, more recent studies indicate involvement of a strong Th17 component $(8,9)$. When antigen presenting cells (APCs) produce TGF- $\beta$, IL-6, and IL-1 $\beta$, naive $\mathrm{CD} 4^{+} \mathrm{T}$ cells differentiate into Th17 cells. This further activates transcription factors ROR $\gamma \mathrm{T}$ and STAT3 and production of Th17 signature cytokines IL-17, IL-22, and IL-21. These Th17 cytokines help recruit and activate additional proinflammatory immune cells such as monocytes and neutrophils to the injured site. Additional studies demonstrated that high-salt intake following an AKI episode reactivates Th17 cells and aggravates tissue fibrosis $(10,11)$. However, the underlying mechanism of sustained Th17 activation in AKI, especially following high-salt intake, is unclear. Furthermore, cellular and molecular mechanisms involved in AKI-to-CKD progression are not completely understood. These basic questions prompted Mehrotra and colleagues to explore the role of the store-operated cal-

Related Article: p. 4951

Conflict of interest: The author has declared that no conflict of interest exists.

cium $\left(\mathrm{Ca}^{2+}\right)$ channel Orai1 in Th17 differentiation and activation. Their data provide compelling evidence that $\mathrm{Ca}^{2+}$ influx by Orai1 is an important mechanism that sustains the Th17-driven inflammatory response after AKI, and drives the AKI-toCKD transition (12).

\section{Store-operated Ca ${ }^{2+}$ channel Orai1}

$\mathrm{Ca}^{2+}$ release-activated $\mathrm{Ca}^{2+}$ (CRAC) channels are membrane proteins that are important for controlling $\mathrm{Ca}^{2+}$ concentration in almost all eukaryotic cells. CRAC gets activated following depletion of endoplasmic reticulum (ER) $\mathrm{Ca}^{2+}$, and this capacitative mechanism is generally referred to as store-operated $\mathrm{Ca}^{2+}$ entry (SOCE). In T cells, SOCE is initiated when an antigen binds to $\mathrm{Fc}$ receptors and activates phospholipase C (PLC). Activation of PLC generates second messenger inositol trisphosphate (IP3), which causes $\mathrm{Ca}^{2+}$ release from ER lumen (13). This reduction in $\mathrm{ER} \mathrm{Ca}^{2+}$ is sensed by stromal interaction molecule (STIM) proteins, which undergo oligomerization and translocate adjacent to the plasma membrane. At these ER-plasma membrane juxtapositions, STIM protein multimers bind to Orail. Orai1, the primary pore-forming subunit of CRAC, exists in two variants: the longer Orai1 $\alpha$ form that contains extra 63 $\mathrm{N}$-terminal amino acids, upstream of the shorter Orai1 $\beta$ (14). The Orai channel proteins (Orai1, 2, and 3) along with STIM proteins (STIM1 and 2) are involved in receptor-regulated $\mathrm{Ca}^{2+}$ signaling in immune cell differentiation, gene regulation, and effector function. For example, STIM1Orail clusters and $\mathrm{Ca}^{2+}$ flux regulate the intensity of nuclear signaling, thereby selectively recruiting different $\mathrm{Ca}^{2+}$ dependent transcription factors (15). Mutations in ORAI1 and STIM1 genes result in defective $\mathrm{Ca}^{2+}$ signaling in $\mathrm{T}$ cells and are associated with impaired $\mathrm{T}$ cell activation and severe combined immunodeficiency in humans, reflecting their importance in several autoimmune and inflammatory diseases (16-18). 
A

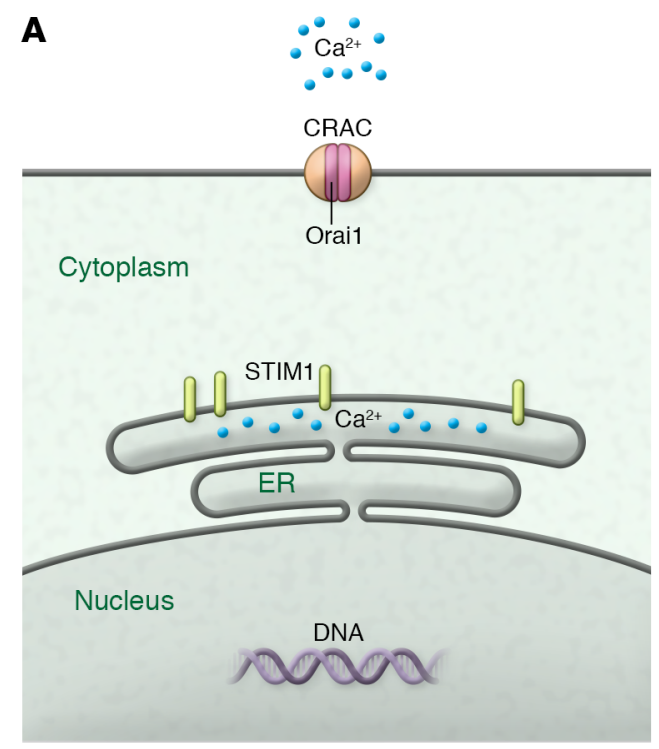

CD4 $+\mathrm{T}$ cell $\downarrow \dot{\mathrm{Ca}^{2+}}$

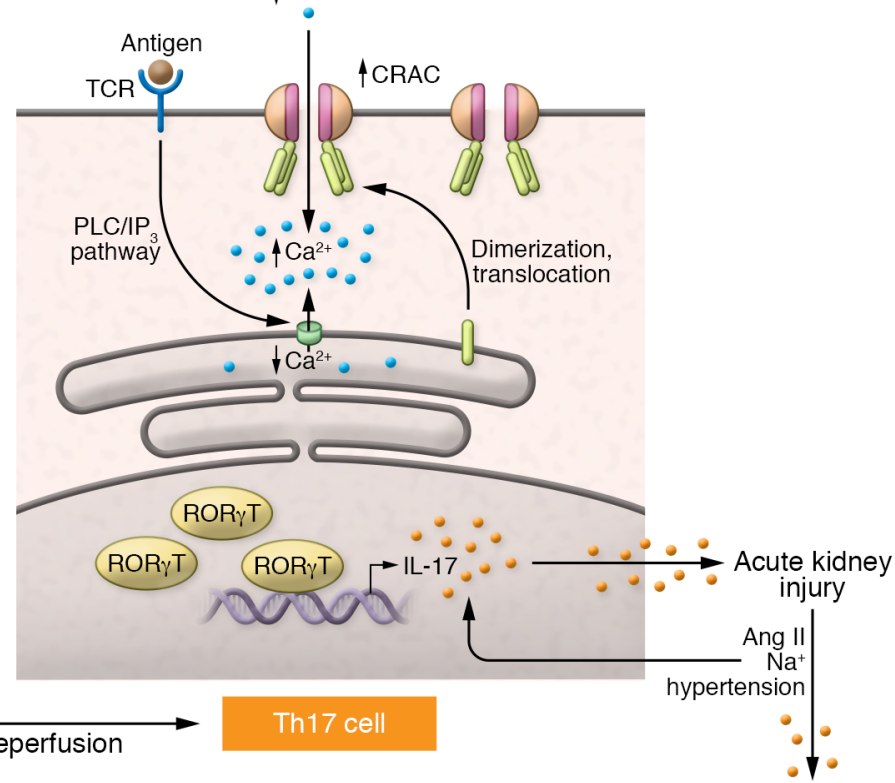

Chronic kidney disease

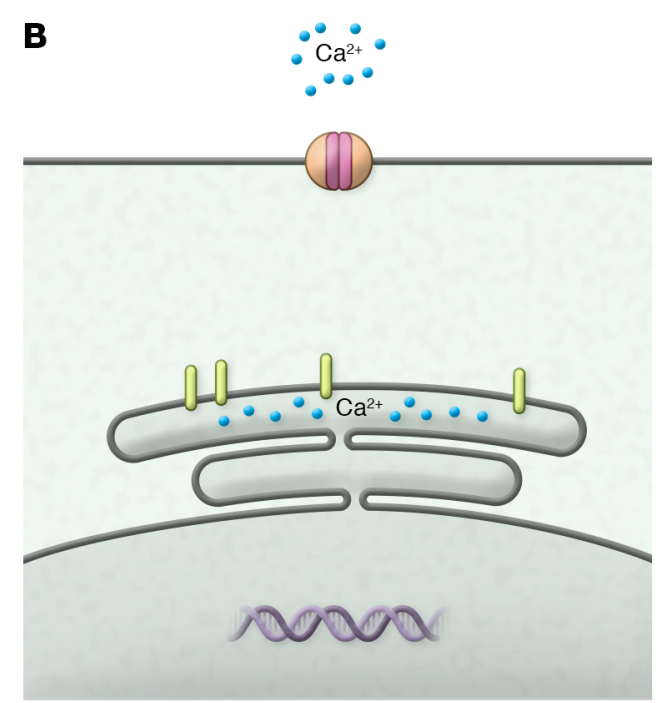

$\mathrm{CD}^{+} \mathrm{T}$ cell

Ischemia reperfusion

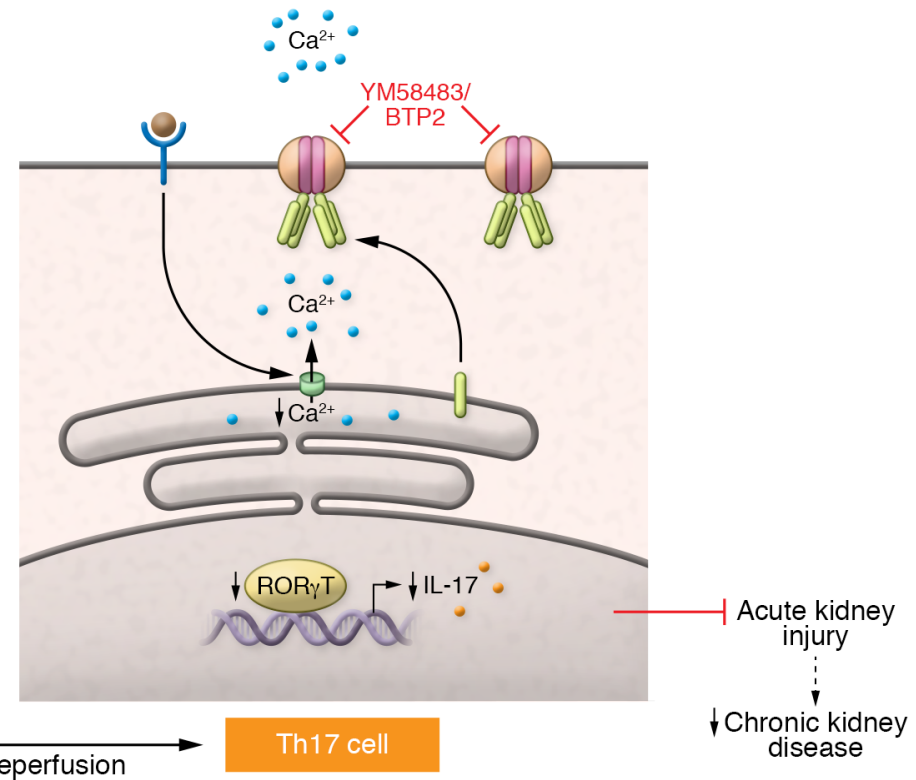

Th17 cell disease

Figure 1. Major events during Orai1-mediated Th17 differentiation in AKI. (A) In resting CD4+ ${ }^{+}$cells, Orai1 is in a closed confirmation and Ca ${ }^{2+}$ is confined to the ER lumen. Following IR, Orai1 expression increases. With subsequent increase in intracellular $\mathrm{Ca}^{2+}$, ROR $\gamma \mathrm{T}$ activity and IL-17 production drives Th17 differentiation and AKI pathogenesis. Simultaneously, antigen binding to the TCR triggers ER Ca ${ }^{2+}$ release, likely via the PLC/IP3 pathway. STIM1, sensing loss of ER $\mathrm{Ca}^{2+}$, translocates to the plasma membrane and activates CRAC protein, resulting in Orai1 opening. Exposure to high Ang II and $\mathrm{Na}^{+}$following AKI reactivates the Th17 response in predominantly Orai1 $1^{+}$cells and sustains inflammation to drive AKI to CKD. (B) CRAC inhibitors, such as YM58483/ BTP2, block $\mathrm{Ca}^{2+}$ influx through Orai1 and may protect from AKI and limit the AKI-to-CKD transition.

Orai1, Ca ${ }^{2+}$ channeling, and Th17 response during AKI

In this issue of the JCI, Mehrotra et al. (12) tested the hypothesis that Orail participates in the activation of Th17 cells and influences the course of renal injury (Figure 1). They initially conducted a series of in vitro and in vivo studies to explore the mechanistic role of Orai1 in Th17 differentiation, AKI pathogenesis, and AKI-to-CKD progression in rats. They then extended their findings to peripheral blood mononuclear cells (PBMCs) from critically ill ICU patients who developed AKI. The authors reported a significant expansion of Orai1-expressing $\mathrm{CD}^{+} \mathrm{T}$ cells 48 hours after IR, which was restricted to IL-17-expressing cells. Interestingly, Orai1 expression remained elevated in post-AKI CD4 ${ }^{+} \mathrm{T}$ cells for up to a week, while Th17 response returned to baseline. Based on these observations, the authors speculated that sustained Orai1 expression in post-AKI CD $4^{+} \mathrm{T}$ cells boosts Th17 reac- 
tivation to a subsequent insult. In fact, in vitro stimulation of post-AKI CD $4^{+} \mathrm{T}$ cells with angiotensin II (Ang II) and sodium $\left(\mathrm{Na}^{+}\right)$increased intracellular $\mathrm{Ca}^{2+}, \mathrm{ROR} \gamma \mathrm{T}$ activity, and IL-17 (mRNA and protein) expression. These results were substantiated by in vivo AKI-to-CKD studies in rats where high-salt administration after IR aggravated chronic renal inflammation, fibrosis, and impaired renal function. Importantly, SOCE inhibitor YM58483, in the presence or absence of high salt, attenuated IR, and glycerol induced Th17 response and renal inflammation and conserved renal structure. In vitro, translational studies on PBMCs from critically ill patients showed increased frequency of ORAI1-expressing cells as well as Th17 cells that further increased under high-salt conditions. These responses were blocked by YM58483, suggesting potential therapeutic applicability of ORAI1 targeting in $\mathrm{AKI}$ and AKI-to-CKD progression in humans.

CRAC-mediated, short-term increase in intracellular $\mathrm{Ca}^{2+}$ is critical for the stable $\mathrm{T}$ cell and APC interactions seen during the formation of an immunological synapse, whereas long-term elevated $\mathrm{Ca}^{2+}$ levels are required to achieve $\mathrm{T}$ cell effector functions (19). Intracellular $\mathrm{ER} \mathrm{Ca}^{2+}$ efflux and CRAC-mediated influx have also been implicated in apoptosis and other related cell death mechanisms in T cells (20). The exciting findings by Mehrotra and colleagues implicate Orai1-mediated $\mathrm{Ca}^{2+}$ channeling as a key mechanism for Th17 differentiation and inflammatory response during AKI, and possibly during AKI-to-CKD transition. In a recent study, $\mathrm{Ca}^{2+}$ signaling was found to control pathogenic Th17-driven inflammation by regulating mitochondrial function, suggesting additional mechanisms are involved in Th17 responses (21). Another recent study, by Mai et al., found a protective effect of Orail blockade in a model of high-fat dietinduced interstitial fibrosis in ApoE-deficient mice, as well as in a model of unilateral ureteral obstruction (UUO) kidney injury in mice (22). Thus, Th17 cell-specific blocking of Orai1 could provide a unique therapeutic opportunity for targeting AKI and ameliorating the effect of a high-salt diet in AKI-to-CKD transition in humans. Furthermore, Th17 targeting in other kidney diseases where the Th17 response is active, such as proliferative glomerulonephritis, could have beneficial effects. However, Orai1 blockade can lead to impairment of $\mathrm{T}$ cell receptor-mediated (TCR-mediated) homeostatic $\mathrm{T}$ cell functions, and should be considered while translating these finding to humans. Additionally, the current study noted a significant increase in creatinine clearance in YM58483-treated rats at the end of the study; however, the authors didn't provide a context to relate clearance to kidney function, or explore long-term consequences of the compound (12). Additionally, Orai1 has been described in CD8 ${ }^{+}$ cells, B cells, NK cells, macrophages, and vascular cells. Recent studies indicate that regulatory Th17 (Treg Th17) cells, distinct from traditional proinflammatory Th17 cells, play an important role in autoimmune diseases. Therefore, it is pertinent to decipher the role of Orai1 in non-T cells, as well as in regulatory $\mathrm{T}$ cells, and the more recently identified double-negative (DN) $\mathrm{T}$ cells, for developing novel inhibitors that can specifically inhibit Th17 Orai1. Although the authors did not observe any change in other Orai proteins (Orai2 and 3) in Th17 cells in their present study, a careful examination of the role of those proteins in renal Treg cells and DN T cells, which predominantly drive antiinflammatory responses during early injury and possibly in the repair and regeneration processes after AKI, will be important $(23,24)$. Furthermore, it is crucial to investigate renal $\mathrm{T}$ cells from AKI patients and compare the responses observed by the authors in PBMCs from ICU patients.

\section{Concluding remarks}

The present findings by Mehrotra et al. highlight Orai1 as a novel mechanism in Th17-driven inflammation with significant therapeutic potential for targeting AKI, AKIto-CKD progression, and other Th17-driven inflammatory and autoimmune diseases. However, due to widespread occurrence of Orai1 in multiple cell types and its role in homeostatic $\mathrm{Ca}^{2+}$ signaling, blocking Orai1 will require extensive future explorations, especially in humans, to develop the most promising and safe therapeutic strategy. Alternatively, modulating Orai1 function indirectly through targeting $\mathrm{Ca}^{2+} / \mathrm{cAMP}$ signaling, which inactivates Orai1 at the end of a signaling loop, may address specificity and circumvent potential adverse effects associated with direct Orai1 blocking (25). Finally, employment of novel technologies, such as single-cell RNA sequencing, T cellspecific Orai1 knockout mice, and CRISPR/ Cas9 (26), will greatly enhance our understanding of the biological roles of Orail and assist in the development of novel inhibitors to treat AKI-to-CKD progression.

\section{Acknowledgments}

The author acknowledges support from NIH grants R01DK111209 and R01DK104662.

Address correspondence to: Sanjeev Noel, 720 Rutland Avenue, Ross 970, Baltimore, Maryland 21205, USA. Phone: 443.287.2703; Email: snoel6@jhmi.edu.

1. Hoste EAJ, et al. Global epidemiology and outcomes of acute kidney injury. Nat Rev Nephrol. 2018;14(10):607-625.

2. Jang HR, Rabb H. Immune cells in experimental acute kidney injury. Nat Rev Nephrol. 2015;11(2):88-101

3. Lee SA, Noel S, Sadasivam M, Hamad ARA, Rabb H. Role of immune cells in acute kidney injury and repair. Nephron. 2017;137(4):282-286.

4. Rabb H, et al. Pathophysiological role of $\mathrm{T}$ lymphocytes in renal ischemia-reperfusion injury in mice. Am J Physiol Renal Physiol. 2000;279(3):F525-F531.

5 . Burne MJ, et al. Identification of the CD4(+) $\mathrm{T}$ cell as a major pathogenic factor in ischemic acute renal failure. JClin Invest. 2001;108(9):1283-1290.

6. Hirahara K, Nakayama T. CD4+ T-cell subsets in inflammatory diseases: beyond the Th1/Th2 paradigm. Int Immunol. 2016;28(4):163-171.

7. Yokota N, Burne-Taney M, Racusen L, Rabb H. Contrasting roles for STAT4 and STAT6 signal transduction pathways in murine renal ischemia-reperfusion injury. Am J Physiol Renal Physiol. 2003;285(2):F319-F325.

8. Li L, et al. IL-17 produced by neutrophils regulates IFN-gamma-mediated neutrophil migration in mouse kidney ischemia-reperfusion injury. J Clin Invest. 2010;120(1):331-342.

9. Lee JW, et al. Transcriptional modulation of the T helper 17/interleukin 17 axis ameliorates renal ischemia-reperfusion injury. Nephrol Dial Transplant. 2019;34(9):1481-1498.

10. Mehrotra P, Collett JA, Gunst SJ, Basile DP. Th17 cells contribute to pulmonary fibrosis and inflammation during chronic kidney disease progression after acute ischemia. Am J Physiol Regul Integr Comp Physiol. 2018;314(2):R265-R273.

11. Mehrotra P, Patel JB, Ivancic CM, Collett JA, Basile DP. Th-17 cell activation in response to high salt following acute kidney injury is associated with progressive fibrosis and attenuated by AT-1R antagonism. Kidney Int. 2015;88(4):776-784.

12. Mehrotra P, Sturek M, Neyra JA, Basile DP. Calcium channel Orai1 promotes lymphocyte IL-17 expression progressive kidney injury. J Clin 
Invest. 2019;129(11):4951-4961.

13. Feske S, Skolnik EY, Prakriya M. Ion channels and transporters in lymphocyte function and immunity. Nat Rev Immunol. 2012;12(7):532-547.

14. Zhang X, Gueguinou M, Trebak M. StoreIndependent Orai Channels Regulated by STIM. In: Kozak JA, Putney JW Jr., eds. Calcium Entry Channels in Non-Excitable Cells. Boca Raton, Florida, USA: CRC Press/Taylor \& Francis; 2018:197-214.

15. Lin YP, Bakowski D, Mirams GR, Parekh AB. Selective recruitment of different $\mathrm{Ca}^{2+}$-dependent transcription factors by STIM1-Orai1 channel clusters. Nat Commun. 2019;10(1):2516.

16. McCarl CA, et al. ORAI1 deficiency and lack of store-operated Ca2+ entry cause immunodeficiency, myopathy, and ectodermal dysplasia. J Allergy Clin Immunol. 2009;124(6):1311-1318.e7. 17. Fuchs $\mathrm{S}$, et al. Antiviral and regulatory $\mathrm{T}$ cell immunity in a patient with stromal interaction molecule 1 deficiency. J Immunol. 2012;188(3):1523-1533.

18. Feske S, et al. Severe combined immunodeficiency due to defective binding of the nuclear factor of activated T cells in T lymphocytes of two male siblings. Eur J Immunol. 1996;26(9):2119-2126.

19. Feske S. Calcium signalling in lymphocyte activation and disease. Nat Rev Immunol. 2007;7(9):690-702.

20. Kim KD, Srikanth S, Yee MK, Mock DC, Lawson GW, Gwack Y. ORAI1 deficiency impairs activated T cell death and enhances T cell survival. J Immunol. 2011;187(7):3620-3630.

21. Kaufmann U, Kahlfuss S, Yang J, Ivanova E, Koralov SB, Feske S. Calcium signaling controls pathogenic Th17 cell-mediated inflammation by regulating mitochondrial function. Cell Metab. 2019;29(5):1104-1118.e6.
22. Mai X, et al. Blockade of Orai1 store-operated calcium entry protects against renal fibrosis. J Am Soc Nephrol. 2016;27(10):3063-3078.

23. Gandolfo MT, et al. Foxp3+ regulatory T cells participate in repair of ischemic acute kidney injury. Kidney Int. 2009;76(7):717-729.

24. Martina MN, et al. Double-negative $\alpha \beta \mathrm{T}$ cells are early responders to AKI and are found in human kidney. J Am Soc Nephrol. 2016;27(4):1113-1123.

25. Zhang $X$, et al. A calcium/cAMP signaling loop at the ORAI1 mouth drives channel inactivation to shape NFAT induction. Nat Commun. 2019;10(1):1971.

26. Noel S, Lee SA, Sadasivam M, Hamad ARA, Rabb H. KEAP1 editing using CRISPR/ Cas9 for therapeutic NRF2 activation in primary human T lymphocytes. J Immunol. 2018;200(5):1929-1936. 